\title{
A preliminary study on the psychosocial impact of COVID-19 lockdown in post-bariatric surgery women: the importance of eating behavior, health care access, and social support
}

\author{
Sílvia Félix ${ }^{1}$ (D) Marta de Lourdes ${ }^{1}$ (D) - Inês Ribeiro ${ }^{1} \cdot$ Bruna Cunha $^{1} \cdot$ Sofia Ramalho ${ }^{1}$ (I) - Ana R. Vaz ${ }^{1}$ (D) \\ Paulo P. P. Machado ${ }^{1}$ (D) Eva Conceição ${ }^{1}$ (D)
}

Accepted: 19 February 2021 / Published online: 28 February 2021

(C) Springer Science+Business Media, LLC, part of Springer Nature 2021

\begin{abstract}
This study aims to characterize the psychosocial impact of COVID-19 lockdown for post-bariatric surgery ( $\geq$ 36 months) women and its association with disordered eating and psychological distress. The medium to longtime follow up is a period of increased susceptibility for poorer weight outcomes which might be triggered by the lockdown. Twenty-four participants responded to an online questionnaire and a telephone interview. About half ( $n=14 ; 58.3 \%$ ) reported perceived weight gain during the lockdown, $13(54.1 \%)$ limited access to social support, and $12(50 \%)$ limited access to medical care. Co-habiting with a higher number of persons during lockdown was associated with fewer difficulties in dealing with emotionally activating situations, less fear of gaining weight, less fear of losing control over eating, and less disordered eating. The global perceived psychosocial impact of lockdown was significantly correlated with difficulties in dealing with emotionally activating situations and stress symptoms. Results highlight the need to monitor post-bariatric patients, facilitate health care access, and promote social support during the lockdown period.
\end{abstract}

Keywords Bariatric surgery · COVID-19 lockdown · Psychosocial impact · Disordered eating behavior · Psychological distress

\author{
Eva Conceição \\ econceicao@psi.uminho.pt \\ Sílvia Félix \\ silviafelix@psi.uminho.pt \\ Marta de Lourdes \\ martamagalhaeslourdes@outlook.com \\ Inês Ribeiro \\ nesnribeiro@gmail.com \\ Bruna Cunha \\ brunacunha@sapo.pt \\ Sofia Ramalho \\ sofia.mm.ramalho@gmail.com \\ Ana R. Vaz \\ anavaz@psi.uminho.pt \\ Paulo P. P. Machado \\ pmachado@psi.uminho.pt \\ 1 School of Psychology, University of Minho, Campus Gualtar, \\ 4710-057 Braga, Portugal
}

\section{Introduction}

The lockdown due to Coronavirus disease (COVID-19) severely impacted multiple domains of people's lives, such as daily routines, employment/financial income, social/family interaction and support, access to food, and access to health care. Furthermore, recent studies focused on the psychological impact of COVID-19 found an increase in stress, anxiety, depression, post-traumatic symptoms, frustration, confusion, uncertainty, fear, and anger in the general population (Brooks et al., 2020; Serafini et al., 2020; de Almeida et al., 2020). Nevertheless, increased attention should be devoted to patients who are particularly predisposed to experience deterioration in their psychological status and/or inaccessibility to adequate treatment (Yao, Chen, \& Xu, 2019). Specifically, in the eating disorders field, particular concerns have been raised about the process involving health care access and the potential increase of disordered eating behaviors, such as grazing, binge eating, and emotional eating (Fernández-Aranda et al., 2020; Weissman, Bauer, \& 
Thomas, 2020). In Portugal, a community-based study carried out during the lockdown highlighted the presence of the following disordered eating behaviors: meal skipping, grazing, overeating, loss of control over eating, and binge eating episodes (39.2\%) during lockdown (Ramalho et al., 2021).

Disordered eating is particularly problematic for individuals receiving bariatric surgery for weight loss because of its association with poor long-term weight outcomes. The need for continuous care for these patients is a hallmark to prevent the onset of disordered eating behaviors and ultimately weight regain (Conceição \& Goldschmid, 2019). A previous study by Sisto and colleagues (2020) stressed that pre- and post-bariatric surgery patients reported increasing hunger, frequency of snacking, and impulse to eat concerning to the COVID-19 pandemic period. On the other hand, the mental health of bariatric patients is also a preoccupation, particularly relevant during the present critical times. Indeed, the same authors concluded that the pandemic led to increased psychological distress in patients included in a bariatric surgery program, expressed in clinically significant anxiety/ depression symptoms, concern about the pandemic, loneliness, and boredom. Moreover, it was also pointed out a relationship between hunger, snacking, eating impulsivity, and anxiety, stress, and depression symptoms (Sisto et al., 2020). Another study in Portugal compared a group of patients in the medium/long term follow up postbariatric surgery (COVID_19 group) and a group of patients reaching the same follow-up time before the pandemic began (NonCOVID-19 group). The authors found that the COVID-19_Group presented higher weight concern, grazing behavior, negative urgency, less weight loss, and increased weight regain than the NonCOVID-19 group (Conceição et al., 2021). Taken together, these pieces of evidence suggest that the coronavirus outbreak negatively impacted eating behaviors, psychological distress, and weight outcomes in bariatric surgery patients (Sisto et al., 2020; Conceição et al., 2021).

Up to date, no study described the specific needs of post-bariatric surgery patients during the lockdown amid the COVID-19 pandemic, particularly for patients in the medium to long-term follow-up ( $>24-36$ months since surgery) since this is a period of increased susceptibility for poorer weight outcomes (Courcoulas et al., 2018). The present study seeks to characterize the experienced psychosocial impact of the COVID-19 lockdown in Portugal for post-bariatric surgery patients with 36 months of follow-up. Specifically, this study will describe the psychosocial impact experiences in varied life domains. This study also aims to investigate the associations between the psychosocial impact of COVID-19 lockdown, disordered eating behavior and psychological distress.

\section{Methods}

\section{Participants and Procedure}

This cross-sectional study included post-bariatric surgery patients from a central hospital in the North of Portugal enrolled in a larger ongoing longitudinal study with different follow-up times. A total of 35 participants of the longitudinal study had their 36-months follow-up assessment during the first lockdown period imposed by the emergency state (i.e., effected in Portugal between March 18 and May 2, 2020). These participants were contacted during the COVID-19 lockdown and answered an online questionnaire in Google Forms assessing sociodemographic and anthropometric data, disordered eating behavior, and psychological distress. Five to eight weeks after the end of the emergency state, these participants were contacted for a structured telephone-interview with approximately 10 min duration that retrospectively assessed the perceived impact of the imposed lockdown on psychosocial, psychological, weight, and disordered eating behavior aspects. At the beginning of the interview, all participants were asked to respond considering the emergency state period. Only 24 (response rate $=68.6 \%$ ) participants accepted participating in the interview and composed the final sample of this manuscript.

The study was approved by the ethical review committees of the university and the hospital involved. All the participants gave their informed consent and were informed about the aims of the study and the voluntary nature of their participation.

\section{Measures}

\section{Self-Reported Measures Applied in Google Forms}

Sociodemographic and Anthropometric Data. It assessed sex, age, marital status, education level, working status, type and date of surgery, current weight (in $\mathrm{kg}$ ) and height (in $\mathrm{cm}$ ).

Other measures: Depression Anxiety Stress Scales (DASS21) (Pais-Ribeiro, Honrado, \& Leal, 2004; Whiteside et al., 2001); UPPS-P Impulsive Behavior Scale - Negative Urgency Subscale (Whiteside, Lynam, Miller, \& Reynolds, 2005) (assesses the tendency to act rashly under negative emotions); Difficulties in Emotion Regulation Scale (DERS) (Coutinho, Dias, \& Ribeiro, 2009; Gratz \& Roemer, 2008); Eating Disorder Examination Questionnaire (EDE-Q) (Machado et al., 2014; Fairburn \& Beglin, 1994) (assesses disordered eating psychopathology); Three Factor Eating Questionnaire R-21 (TFEQ-R21) (Cappelleri et al., 2009; Duarte, Palmeira, \& Pinto, 2018) (assesses disordered eating); Loss of Control over Eating Scale (LOCES) (Latner, Mond, Kelly, Haynes, \& Hay, 2014).; Repetitive Eating Questionnaire [Rep(eat)-Q] (Conceição, Mitchell, Machado, Vaz, Pinto- Bastos, Ramalho et al., 2017) (assesses grazingeating pattern). 


\section{Structured Telephone-Interview}

Impact on Emotions, Eating, and Weight (IEEW). Four questions were used by the authors to screen the perceived impact of the lockdown on psychological/eating status. "During the lockdown period: (1) Did you find it difficult to deal with situations in which you felt very angry, frustrated, sad or upset?; (2) Did you feel any mood change?; (3) How afraid were you of gaining weight?; and (4) How afraid were you of losing control over eating?". All items were responded on a 4-point Likert scale $(0=$ Nothing; $3=$ Extremely). Two multiple-choice questions asked about perceptions of weight change (i.e., increase, decrease or maintenance), and weight status (i.e., underweight, normal weight, overweight, obesity) during the COVID-19 lockdown period.

Coronavirus Impact Scale (Kaufman \& Stoddard, 2020). The proposed scale by Stoddard (2020) comprises 11 items rated on a 4-points Likert scale that assessed the extent of change experienced due to COVID-19 lockdown in: the respondent daily routines; family income/employment; access to food; access to medical health care; access to mental health treatment; access to social support; experience of stress; stress and discord in the family; personal, immediate/extended family, and close friends diagnosis of COVID-19 and respective symptoms if applicable. The total score was calculated by the sum of participants' answers to all 11 closed-ended questions and correspond to the COVID-19 impact score. There is an additional open-ended item to assess other possible areas affected by COVID-19.

\section{Statistical Analysis}

The IBM $®$ SPSS $®$ Statistics 25 (SPSS Inc., Chicago, IL) was used for data analyses. Descriptive statistics were used to examine the demographic, anthropometric, and psychosocial impact of Coronavirus variables. Pearson (for variables with a normal distribution) and Spearman (for variables with a non-normal distribution) correlation coefficients were performed to identify bivariate associations among the variables under study. The $p$-values of $<.05$ were interpreted as statistically significant. To minimize the type I error due to the high number of correlation tests, only correlations corresponding to medium or large effect sizes were considered meaningful (i.e., $>.40$ for Pearson's $r$ and Spearman's rho) (Cohen, 1988). A post hoc power analysis was conducted using the software $G^{*}$ Power 3.1 and revealed that to a correlation between .42 and .85 (the minimum and maximum correlation coefficient, respectively), with alpha of .005 and 24 participants, the statistical power range from .67 to .99 .

\section{Results}

Participants were 24 post-bariatric surgery ( $\geq 36$ months of follow-up) women. Sociodemographic and anthropometric information is displayed in Table 1. Twelve (50\%) participants perceived their weight status during the lockdown (IEEW) as normal weight, $45.8 \%(n=11)$ as overweight, and only one $(5 \%)$ as obesity. The majority reported perceived weight gain during the lockdown period $(n=14 ; 58.3 \%), 20.8 \%(n=5)$ perceived no weight change, and $20.8 \%(n=5)$ perceived weight loss (IEEW).

Participants reported a total mean score of $9.96(\mathrm{SD}=3.86$, Min $=3$, Max $=20$ ) on the Coronavirus Impact Scale (possible scores range from 0 to 33). The frequencies of response to the individual items are presented in Table 2. Notable percentages of the sample reported moderate to severe impact of COVID-19 lockdown in the following areas: $75 \%(n=18)$ in daily routines; $54.1 \%(n=13)$ in access to extended family and non-family social support; $50 \%(n=12)$ in access to medical care; $33.4 \%(n=8)$ in family income/employment; $25 \%$ $(n=6)$ in access to mental health care; $20.8 \%(n=5)$ in access to food; $20.8 \%(n=5)$ experience of stress related to Coronavirus pandemic; and 20.8\% $(\mathrm{n}=5)$ and stress/discord in the family. Only one (4.2\%) participant was diagnosed with COVID-19 reporting mild symptoms, three (12.5\%) participants had an immediate or extended family member or close friend diagnosed with COVID-19. When openly questioned about other areas affected by Coronavirus pandemic, the most reported were the experience of fear $(n=5,20.8 \%)$, and the extreme reduction of social life events $(n=5,20.8 \%)$.

Table 3 presents the correlations between the total score of the Coronavirus Impact Scale and other psychological and disordered eating behavior variables assessed. Higher scores on the Coronavirus impact scale were linked to higher difficulties in dealing with situations when they felt angry, frustrated, sad, or upset (i.e., emotionally activating situations; IEEW; $\mathrm{r}_{\mathrm{s}}=.45, p=.027$ ), and with higher stress symptoms (DASS-21; $\mathrm{r}_{\mathrm{S}}=.44, p=.030$ ). Co-habiting with a higher number of persons during lockdown was associated with fewer difficulties in dealing with emotionally activating situations (IEEW; $\mathrm{r}_{\mathrm{s}}=-.49, p=.015$ ), less fear of gaining weight (IEEW; $\mathrm{r}_{\mathrm{s}}=-.48, p=.019$ ), less fear of losing control over eating (IEEW; $\mathrm{r}_{\mathrm{s}}=-.56, p=.005$ ), and less disordered eating psychopathology (EDE-Q; $\left.\mathrm{r}_{\mathrm{s}}=-.47, p=.020\right)$. Higher difficulties in dealing with emotionally activating situations during lockdown (IEEW) were linked with more mood changes (IEEW; $\mathrm{r}_{\mathrm{s}}=.75, p<.001$ ), fear of gaining weight (IEEW; $\mathrm{r}$ $\left.{ }_{\mathrm{s}}=.42, p=.040\right)$, depression (DASS-21; $\left.\mathrm{r}_{\mathrm{s}}=.54, p=.007\right)$, anxiety (DASS-21; $\mathrm{r}_{\mathrm{s}}=.55, p=.005$ ), and stress symptoms (DASS-21; $\mathrm{r}_{\mathrm{s}}=.61, p=.002$ ). More mood changes during lockdown (IEEW) were linked with higher depression (DASS-21; $\mathrm{r}_{\mathrm{s}}=.58, p=.003$ ) and anxiety symptoms (DASS$\left.21 ; \mathrm{r}_{\mathrm{s}}=.47, p=.020\right)$. Higher fear of gaining weight during 
Table 1 Participants' sociodemographic characterization

\begin{tabular}{|c|c|c|c|c|}
\hline$N=24$ & $\mathrm{n}(\%)$ & Mean (SD) & Min & Max \\
\hline Age (years) & - & $50.92(12.81)$ & 30 & 71 \\
\hline BMI $\left(\mathrm{kg} / \mathrm{m}^{2}\right)$ & - & $30.64(5.06)$ & 22.49 & 40.72 \\
\hline \multicolumn{5}{|l|}{ Marital status } \\
\hline Single & $4(16.7 \%)$ & - & - & - \\
\hline Married/Cohabiting & $11(45.8 \%)$ & - & - & - \\
\hline Divorced & $6(25 \%)$ & - & - & - \\
\hline Widow & $3(12.5 \%)$ & - & - & - \\
\hline \multicolumn{5}{|l|}{ Educational level } \\
\hline Elementary school & $7(29.2 \%)$ & - & - & - \\
\hline Middle school & $6(25 \%)$ & - & - & - \\
\hline High school & $3(12.5 \%)$ & - & - & - \\
\hline College degree & $8(33.3 \%)$ & - & - & - \\
\hline \multicolumn{5}{|l|}{ Employment status } \\
\hline Employed & $16(66.7 \%)$ & - & - & - \\
\hline Unemployed & $2(8.3 \%)$ & - & - & - \\
\hline Retired & $6(25 \%)$ & - & - & - \\
\hline \multicolumn{5}{|l|}{ Type of surgery } \\
\hline Gastric bypass & $16(66.7 \%)$ & - & - & - \\
\hline Sleeve gastrectomy & $8(33.3 \%)$ & - & - & - \\
\hline Follow-up time since surgery (months) & - & $42.88(4.73)$ & 37 & 51 \\
\hline
\end{tabular}

$B M I=$ Body Mass Index lockdown (IEEW) was associated with a higher fear of losing control over eating (IEEW; $\left.\mathrm{r}_{\mathrm{s}}=.60, p=.002\right)$, and disordered eating behaviors and attitudes (EDE-Q; $\left.\mathrm{r}_{\mathrm{s}}=.56, p=.005\right)$.

Additional correlations were performed between the individual items of the Coronavirus Impact Scale representing change experienced in specific life domains, and psychological and disordered eating behavior (Supplementary Material - Table S1). Results showed that a higher impact on access to mental health care was linked with higher difficulties in dealing with emotionally activating situations (IEEW; $\mathrm{r}_{\mathrm{s}}=.59, p=.002$ ), $\operatorname{mood}$ changes (IEEW; $\mathrm{r}_{\mathrm{s}}=.56, p=.005$ ), and depression symptoms (DASS-21; $\mathrm{r}_{\mathrm{s}}=.47, p=.020$ ). Higher experience of stress related to Coronavirus pandemic was also associated with higher difficulties in dealing with emotionally activating situations (IEEW; $\left.\mathrm{r}_{\mathrm{s}}=.44, p=.034\right)$, depression $\left(\mathrm{r}_{\mathrm{s}}=.45, p=.029\right)$ and anxiety symptoms (DASS-21; $\mathrm{r}_{\mathrm{s}}=.46, p=.023$ ), and emotion dysregulation (DERS; $\left.\mathrm{r}_{\mathrm{s}}=.56, p=.005\right)$.

\section{Discussion}

The present study aimed to characterize the psychosocial impact of the Coronavirus epidemic in post-bariatric surgery patients during the lockdown and its association with psychological status and disordered eating behaviors. Our findings add important knowledge regarding the specific needs of this population during this period. Specifically, a notable percentage of bariatric surgery patients reported perceived weight gain which is of concern since this sample was assessed in a critical follow-up time (36 months since surgery). This is a period of increased susceptibility for poorer weight outcomes (Courcoulas et al., 2018) which might be triggered by the lockdown. Although the perception of weight variations might be different from the real weight change, perceived weight gain on itself may indicate underlying disordered eating attitudes/behaviors making it a target for clinical attention (Haynes, Kersbergen, Sutin, Daly, \& Robinson, 2018). Conversely, these patients benefit from close monitoring and earlier detection of dysfunctional behaviors (Conceição \& Goldschmid, 2019; Pinto-bastos, de Lourdes, Brandão, Machado, \& Conceição, 2019) for which the limited access to health and mental care perceived by $50 \%$ and $25 \%$ of patients, respectively, is of concern. These results are in line with a previous study assessing pre- and post-bariatric patients that found out only one-fifth of patients continuing direct bariatric care (Waledziak et al., 2020).

Our findings show that co-habiting with more persons during lockdown was correlated with fewer difficulties in dealing with emotionally activating situations and less disordered eating behaviors and attitudes. Accordingly, previous literature showed that the perceived support from family, friends, and significant others is thought to be associated with less psychological distress, less disordered eating, and more weight loss post-surgery (Conceição, Fernandes, de Lourdes, Pinto-Bastos, Vaz, Ramalho, 2019). Thus, the perceived difficulty in maintaining a 
Table 2 Descriptive statistics of Coronavirus Impact Scale items assessed during lockdown

\begin{tabular}{|c|c|c|c|c|}
\hline \multirow[t]{2}{*}{ Items } & \multicolumn{4}{|l|}{$\begin{array}{l}\text { Frequencies } \\
\mathrm{n}(\%)\end{array}$} \\
\hline & None & Mild & Moderate & Severe \\
\hline \multicolumn{5}{|l|}{$\begin{array}{l}\text { Rate how much the Coronavirus pandemic has changed your life in } \\
\text { each of the following ways: }\end{array}$} \\
\hline 1 - Routines & $2(8.3 \%)$ & $4(16.7 \%)$ & $6(25 \%)$ & $12(50 \%)$ \\
\hline 2 - Family income/employment & $12(50 \%)$ & $4(16.7 \%)$ & $4(16.7 \%)$ & $4(16.7 \%)$ \\
\hline 3 - Access to food & $14(58.3 \%)$ & $5(20.8 \%)$ & $3(12.5 \%)$ & $2(8.3 \%)$ \\
\hline 4 - Access to medical health care & $4(16.7 \%)$ & $8(33.3 \%)$ & $9(37.5 \%)$ & $3(12.5 \%)$ \\
\hline 5 - Access to mental health treatment & $17(70.8 \%)$ & $1(4.2 \%)$ & $5(20.8 \%)$ & $1(4.2 \%)$ \\
\hline 6 - Access to extended family and non-family social supports & $0(0 \%)$ & $11(45.8 \%)$ & $11(45.8 \%)$ & $2(8.3 \%)$ \\
\hline 7 - Experiences of stress related to coronavirus pandemic & $2(8.3 \%)$ & $11(45.8 \%)$ & $4(16.7 \%)$ & $7(29.2 \%)$ \\
\hline $8-$ Stress and discord in the family & $16(66.7 \%)$ & $3(12.5 \%)$ & $4(16.7 \%)$ & $1(4.2 \%)$ \\
\hline 9 - Personal diagnosis of coronavirus (and symptoms if applicable) & $23(95.8 \%)$ & $1(4.2 \%)$ & $0(0 \%)$ & $0(0 \%)$ \\
\hline $\begin{array}{l}10 \text { - Immediate family member(s) diagnosed with coronavirus } \\
\text { (and symptoms if applicable) }\end{array}$ & $23(95.8 \%)$ & $1(4.2 \%)$ & $0(0 \%)$ & $0(0 \%)$ \\
\hline \multirow{2}{*}{$\begin{array}{l}11 \text { - Extended family member(s) and/or close friends diagnosed with } \\
\text { coronavirus (and symptoms if applicable) }\end{array}$} & $22(91.7 \%)$ & $2(8.3 \%)$ & $0(0 \%)$ & $0(0 \%)$ \\
\hline & \multicolumn{3}{|c|}{ Topic reported } & $\begin{array}{l}\text { Frequency } \\
\mathrm{n}(\%)\end{array}$ \\
\hline \multirow{7}{*}{$\begin{array}{l}12 \text { - Please tell us about any other ways the coronavirus pandemic } \\
\text { has impacted your life }\end{array}$} & \multicolumn{3}{|c|}{ Experience of fear } & $5(20.8 \%)$ \\
\hline & \multicolumn{3}{|c|}{ Extreme reduction in social life } & $5(20.8 \%)$ \\
\hline & \multicolumn{3}{|c|}{ Loss of the job } & $2(8.3 \%)$ \\
\hline & \multicolumn{3}{|c|}{ Self-medication due to lack of medical assistance } & $1(4.2 \%)$ \\
\hline & \multicolumn{3}{|c|}{ Deterioration of mental health due to lack treatment } & $1(4.2 \%)$ \\
\hline & \multicolumn{3}{|c|}{ Increased anxiety } & $1(4.2 \%)$ \\
\hline & \multicolumn{3}{|c|}{ Financial impact } & $1(4.2 \%)$ \\
\hline
\end{tabular}

social support network and good-quality relationships with family members reported in our sample may put these patients at increased risk for poor outcomes.

These results also point to an association between the psychosocial impact experienced during lockdown and difficulties in dealing with emotionally activating situations and with higher stress symptoms. These findings bring further evidence to the previous general recommendations highlighting the importance of attending to the psychological status, facilitate the access to adequate health treatment, and deal with isolation/lack of social support to minimize the COVID-19 impact (Yao et al., 2019; Fernández-Aranda et al., 2020; Weissman et al., 2020).

Regarding the limitations of this investigation, this is a preliminary study with a rather sample size which comprised only of women. Future studies should address the same variables with a larger sample size and be composed of participants of both sexes. Notwithstanding, although these aspects might limit the generalization of results, they also represent a headway toward understanding how the lockdown period might impact bariatric surgery treatment. Secondly, despite weight and height being self-reported, past research supports the clinical relevance of experienced of weight variations (Haynes et al., 2018) and cumulating evidence suggests that bariatric patients self-report their weight reasonably accurately (White, Masheb, BurkeMartindale, Rothschild, \& Grilo, 2007). Third, the questionnaires to assess the perceived psychosocial impact of the imposed lockdown (i.e., IEEW and Coronavirus Impact Scale) are yet to be validated. However, these measures are not intended to assess unobservable psychological constructs but rather they ask directly about the perceived impact of different dimensions of daily-life. Similarly, the correlations should be indicative of the statistical and clinical relevance of our findings. Finally, we used a cross-sectional design that does not allow causality inferences between perceived impact and the psychological aspects evaluated.

\section{Conclusion}

To the best of our knowledge, this is the first study that characterized the psychosocial impact of the COVID-19 lockdown period and its association with psychological status and disordered eating behavior in bariatric surgery patients undergoing critical follow-up times since surgery. The findings alert 
Table 3 Correlations among variables under study

\begin{tabular}{|c|c|c|c|c|c|c|c|c|c|c|c|c|c|c|c|c|}
\hline & 1 & 2 & 3 & 4 & 5 & 6 & 7 & 8 & 9 & 10 & 11 & 12 & 13 & 14 & 15 & 16 \\
\hline 1 - Coronavirus Impact Scale & - & & & & & & & & & & & & & & & \\
\hline $2-\mathrm{BMI}$ & $-.10^{\mathrm{a}}$ & - & & & & & & & & & & & & & & \\
\hline $\begin{array}{l}3 \text { - Number of persons in co-- } \\
\text { habitation }\end{array}$ & .23 & -.28 & - & & & & & & & & & & & & & \\
\hline $\begin{array}{l}\text { 4-IEEW Difficulties in dealing } \\
\text { with situations }\end{array}$ & $.45^{*}$ & .14 & $-.49 *$ & - & & & & & & & & & & & & \\
\hline 5 - IEEW Mood changes & .39 & .18 & .18 & $.75^{* * * *}$ & - & & & & & & & & & & & \\
\hline $\begin{array}{l}6 \text { - IEEW Fear of gaining } \\
\text { weight }\end{array}$ & .04 & $.45^{*}$ & $-.48^{*}$ & $.42 *$ & .09 & - & & & & & & & & & & \\
\hline $\begin{array}{l}7 \text { - IEEW Fear of losing control } \\
\text { over eating }\end{array}$ & .15 & .14 & $-.56 * *$ & .21 & .01 & $.60 * *$ & - & & & & & & & & & \\
\hline 8 - DASS-21 Depression & .37 & .24 & -.29 & $.54 * *$ & $.58 * *$ & .09 & .06 & - & & & & & & & & \\
\hline 9 - DASS-21 Anxiety & .30 & .29 & -.25 & $.55^{* *}$ & $.47 *$ & .29 & .05 & $.85 * * *$ & - & & & & & & & \\
\hline 10 - DASS-21 Stress & $.44 *$ & .14 & -.21 & $.61 * *$ & .35 & .34 & .06 & $.72 * * *$ & $.85^{* * * *}$ & - & & & & & & \\
\hline 11 - DERS Total & $.10^{\mathrm{a}}$ & $.37^{\mathrm{a}}$ & -.21 & .29 & .09 & .51 & .23 & .34 & $.46^{*}$ & .38 & - & & & & & \\
\hline 12 - UPPS-P NU & $.05^{\mathrm{a}}$ & $.20^{\mathrm{a}}$ & -.25 & .18 & -.03 & .21 & .17 & $.48 *$ & $.45^{*}$ & $.45^{*}$ & $.42 *^{\mathrm{a}}$ & - & & & & \\
\hline 13 - EDE-Q Total & $-.08^{\mathrm{a}}$ & $.42 *$ & $-.47 *$ & .27 & .18 & $.56 * *$ & .35 & $.44 *$ & .36 & .25 & $.51 *^{\mathrm{a}}$ & $.56^{* * *^{\mathrm{a}}}$ & - & & & \\
\hline 14 - TFEQ-R21 Total & $.25^{\mathrm{a}}$ & $.01^{\mathrm{a}}$ & .08 & -.13 & .01 & -.34 & -.03 & $.42 *$ & .40 & .21 & $.08^{\mathrm{a}}$ & $.21^{\mathrm{a}}$ & $-.01^{\mathrm{a}}$ & - & & \\
\hline 15 - LOCES Total & .19 & .12 & .12 & -.22 & -.25 & -.07 & -.04 & .15 & .26 & .20 & .27 & $.53 * *$ & .12 & $.49 *$ & - & \\
\hline 16 - Rep(eat)-Q Total & .25 & .07 & .32 & -.39 & -.33 & -.19 & -.03 & .05 & .05 & .01 & .06 & $.43 *$ & .23 & $.55^{* *}$ & $.83^{* * * *}$ & - \\
\hline
\end{tabular}

a Pearson's Correlation coefficients; all the other are Spearman's Correlation coefficients

$* p<.05, * * p<.01, * * * p<.001$

The values highlighted in bold correspond to moderate or strong effect sizes (i.e., >. 40 for Pearson's and Spearman's Correlations)

$B M I=$ Body Mass Index; IEEW = Impact on Emotions, Eating, and Weight; DASS-21 = Depression Anxiety Stress Scales; DERS Total = Difficulties in Emotion Regulation Scale - Score Total; UPPS-P NU = UPPS-P Impulsive Behavior Scale - Negative Urgency Subscale; EDE- $Q$ Total = Eating Disorder Examination Questionnaire - Score Total; TFEQ-R21 Total= Three Factor Eating Questionnaire R-21 - Score Total; LOCES Total=Loss of Control over Eating Scale - Score Total; Rep(eat)-Q Total = Repetitive Eating Questionnaire - Score Total

clinicians in the field of the need for close monitoring of these patients and the importance of facilitating health care access and promoting social support during the lockdown period.

Supplementary Information The online version contains supplementary material available at https://doi.org/10.1007/s12144-021-01529-6.

Authors' Contribution All authors contributed to the study conceptualization and to the development/design of methodology. Data collection were complete by Marta de Lourdes, Sílvia Félix, Bruna Cunha, and Inês Ribeiro. Material preparation and formal analysis were performed by Eva Conceição, Marta de Lourdes, and Sílvia Félix. The first draft of the manuscript was written by Eva Conceição and Sílvia Félix. All authors reviewed/comment previous versions and approved the final manuscript.

Funding This study was conducted at the Psychology Research Centre (CIPsi/UM) School of Psychology, University of Minho, supported by the Foundation for Science and Technology (FCT) through the Portuguese State Budget (UIDB/01662/2020), by grants to Eva Conceição (IF/01219/2014 and POCI-01-0145-FEDER-028209), and Marta de Lourdes (SFRH/BD/146470/2019). Dr. Machado was supported, in part, by a Portuguese Foundation for Science and Technology grant (POCI-01-0145-FEDER-028145). The funding body had no role in the design, collection, analysis, and interpretation of data; the writing of the manuscript; or the decision to submit the manuscript for publication.
Data Availability The datasets generated during and/or analyzed during the current study are available from the corresponding author on reasonable request.

\section{Declarations}

Ethics Approval All procedures performed were in accordance with the ethical standards of the university and the hospital research committees, and with the 1964 Helsinki declaration and its later amendments. This study was approved by the ethical review committees of the University of Minho and the hospital involved.

Informed Consent Informed consent was obtained from all individual participants included in the study.

Conflicts of Interest/Competing Interests The authors have no relevant financial or non-financial interests to disclose.

\section{References}

Brooks, S. K., Webster, R. K., Smith, L. E., Woodland, L., Wessely, S., Greenberg, N., \& Rubin, G. J. (2020). The psychological impact of quarantine and how to reduce it: Rapid review of the evidence. Lancet, 395(10227), 912-920. 
Cappelleri, J. C., Bushmakin, A. G., Gerber, R. A., Leidy, N. K., Sexton, C. C., Lowe, M. R., et al. (2009). Psychometric analysis of the Three-Factor Eating Questionnaire-R21: results from a large diverse sample of obese and non-obese participants. International Journal of Obesity, 33(6), 611-620. Available from: http://www.ncbi.nlm. nih.gov/pubmed/19399021.

Cohen, J. (1988). Statistical power analysis for the behavioral sciences. Hillsdale, editor. New Jersey: Lawrence Erlbaum Associates.

Conceição, E., \& Goldschmid, A. (2019). Disordered eating after bariatric surgery: Clinical aspects, impact on outcomes, and intervention strategies. Current Opinion in Psychiatry, 32(6), 504-509.

Conceição, E. M., Mitchell, J. E., Machado, P. P. P., Vaz, A. R., PintoBastos, A., Ramalho, S., Brandão, I., Simões, J. B., de Lourdes, M., \& Freitas, A. C. (2017). Repetitive eating questionnaire [Rep(eat)Q]: Enlightening the concept of grazing and psychometric properties in a Portuguese sample. Appetite, 117, 351-358.

Conceição, E. M., Fernandes, M., de Lourdes, M., Pinto-Bastos, A, Vaz, A. R., Ramalho, S. (2019). Perceived social support before and after bariatric surgery: Association with depression, problematic eating behaviors, and weight outcomes. Eat weight Disord - stud anorexia, Bulim Obes, 25(3), 679-692. Available from: https://doi.org/10. 1007/s40519-019-00671-2, 2020.

Conceição, E., de Lourdes, M., Ramalho, S., Félix, S., Pinto-Bastos, A., Vaz, A. R. (2021). Eating behaviors and weight outcomes in bariatric surgery patients amidst COVID-19. Surgery for Obesity and Related Diseases. Accepted publication.

Courcoulas, A. P., King, W. C., Belle, S. H., Berk, P., Flum, D. R., Garcia, L., Gourash, W., Horlick, M., Mitchell, J. E., Pomp, A., Pories, W. J., Purnell, J. Q., Singh, A., Spaniolas, K., Thirlby, R., Wolfe, B. M., \& Yanovski, S. Z. (2018). Seven-year weight trajectories and health outcomes in the longitudinal assessment of bariatric surgery (LABS) study. JAMA Surgery, 153(5), 427-434.

Coutinho, J., Dias, P. C., \& Ribeiro, E. (2009). The Portuguese version of the difficulties in emotion regulation scale and its relationship with psychopathological symptoms Versão Portuguesa da Escala de Dificuldades de Regulação Emocional e sua relação com sintomas psicopatológicos. Revista Psiquiatry Clinica, 37(4), 145-151.

de Almeida, T. C., Heitor, M. J., Santos, O., Costa, A., Virgolino, A., Rasga, C., et al. (2020). Saúde mental em tempos de pandemia - SMCOVID-19: relatório final. Lisbon: Instituto Nacional de Saúde Doutor Ricardo Jorge, IP.

Duarte, P. A. S., Palmeira, L., Pinto, J. (2018). The Three-Factor Eating Questionnaire-R21: a confirmatory factor analysis in a Portuguese sample. Eat Weight Disord - Stud Anorexia Bulim Obes, 25(1), 247 256. Available from: https://doi.org/10.1007/s40519-018-0561-7.

Fairburn, C. G., \& Beglin, S. J. (1994). Assessment of eating disorders: Interview or self-report questionnaire? Intnational Journal of Eating Disorders, 16, 4.

Fernández-Aranda, F., Casas, M., Claes, L., Bryan, D. C., Favaro, A., Granero, R., Gudiol, C., Jiménez-Murcia, S., Karwautz, A., le Grange, D., Menchón, J. M., Tchanturia, K., \& Treasure, J. (2020). COVID-19 and implications for eating disorders. European Eating Disorders Review, 28, 239-245.

Gratz, K. L., \& Roemer, L. (2008). Multidimensional assessment of emotion regulation and Dysregulation: Development, factor structure, and initial validation of the difficulties in emotion regulation scale. Journal of Psychopathology and Behavioral Assessment, 30(4), 41-54.

Haynes, A., Kersbergen, I., Sutin, A., Daly, M., \& Robinson, E. (2018). A systematic review of the relationship between weight status perceptions and weight loss attempts, strategies, behaviours and outcomes. Obesity Reviews, 19, 347-363.
Kaufman, J., Stoddard, J. (2020). Coronavirus impact scale.

Latner, J. D., Mond, J. M., Kelly, M. C., Haynes, S. N., \& Hay, P. J. (2014). The loss of control over eating scale : Development and psychometric evaluation. The International Journal of Eating Disorders, 47, 647659.

Machado, P. P. P., Martins, C., Vaz, A.R., Conceição, E., Bastos, A. P., Gonçalves, S. (2014). Eating disorder examination questionnaire: Psychometric properties and norms for the Portuguese population. European Eating Disorders Review, 22(6), 448-453. Available from: https://doi.org/10.1002/erv.2318.

Pais-Ribeiro, J. L., Honrado, A., \& Leal, I. (2004). Contribuição Para O Estudo Da Adaptação Portuguesa Das Escalas De Ansiedade, Depressão E Stress ( Eads ) De 21 Itens De Lovibond E Lovibond. Psicol Saúde Doenças, 5(2), 229-239.

Pinto-bastos, A., de Lourdes, M., Brandão, I., Machado, P. P. P., \& Conceição, E. M. (2019). Weight loss trajectories and psychobehavioral predictors of outcome of primary and reoperative bariatric surgery: A 2-year longitudinal study. Surgery for Obesity and Related Diseases, 15, 1104-1112.

Ramalho, S., Trovisqueira, A., de Lourdes, M., Gonçalves, S., Ribeiro, I., Vaz, A. R., et al. (2021). The impact of COVID-19 lockdown on disordered eating behaviors: The mediation role of psychological distress. Eat Weight Disord - Stud Anorexia, Bulim Obes. Accepted publication. Available from: https://doi.org/10.1007/ s40519-021-01128-1.

Serafini, G., Parmigiani, B., Amerio, A., Aguglia, A., Sher, L., \& Amore, M. (2020). The psychological impact of COVID-19 on the mental health in the general population. QJM An Int J Med, 113(8), 529535.

Sisto, A., Vicinanza, F., Tuccinardi, D., Watanabe, M., Francesca, I., D'Alessio, R., et al. (2020). The psychological impact of COVID 19 pandemic on patients included in a bariatric surgery program. Eat Weight Disord - Stud Anorexia, Bulim Obes. Available from: https:// doi.org/10.1007/s40519-020-00988-3.

Waledziak, M., Rózanska-Waledziak, A., Pedziwiatr, M., Szeliga, J., Proczko-stepaniak, M., Stefura, T., et al. (2020). Bariatric surgery during COVID-19 pandemic from patients ' point of view - The results of a National Survey. Journal of Clinical Medicine, 9, 1-13.

Weissman, R. S., Bauer, S., Thomas, J. J. (2020) Access to evidencebased care for eating disorders during the COVID-19 crisis. International J Eat Disord, 53(5), 639-646. Available from: https://doi.org/10.1002/eat.23279.

White, M. A., Masheb, R. M., Burke-Martindale, C., Rothschild, B., \& Grilo, C. M. (2007). Accuracy of self-reported weight among bariatric surgery candidates: The influence of race and weight cycling. Obesity, 15, 2761-2768.

Whiteside, S. P., Lynam, D. R., Miller, J. D., Reynolds, S. K., Duarte, C., Gouveia, J. P, et al. (2001). Reliability testing of a children's version of the eating attitude test. Journal of Counseling Psychology [Internet] 10(3), 351-358. Available from: http://www.sciencedirect.com.spot. lib.auburn.edu/science/article/pii/S0890856709658020.

Whiteside, S. P., Lynam, D., Miller, J., \& Reynolds, S. (2005). Validation of the UPPS impulsive behavior scale: A four factor model of impulsivity. European Journal of Personality, 19, 559-574.

Yao, H., Chen, J., Xu, Y. (2019). Patients with mental health disorders in the COVID-19 epidemic. The Lancet Psychiatry, 7(4):e21. Available from: https://doi.org/10.1016/S2215-0366(20)30090-0.

Publisher's Note Springer Nature remains neutral with regard to jurisdictional claims in published maps and institutional affiliations. 